\title{
Optimum Coil-System Layout for Magnet-Driven Superconducting Magnetic Density Separation
}

\author{
J. J. Kosse ${ }^{\circledR 1}$, M. Dhallé ${ }^{1}$, G. Tomás ${ }^{\circledR 1}$, P. C. Rem², H. J. M. ter Brake ${ }^{1}$, and H. H. J. ten Kate ${ }^{\circledR 1}$ \\ ${ }^{1}$ Faculty of Science and Technology, University of Twente, 7500 AE Enschede, The Netherlands \\ ${ }^{2}$ Faculty of Civil Engineering and Geosciences, Delft University of Technology, 2628 CD Delft, The Netherlands
}

\begin{abstract}
This article discusses the optimum layout of coils of a superconducting magnet system for magnetic density separation (MDS). MDS is a novel separation technology that combines a vertical magnetic field gradient with a ferrofluid to separate mixtures of non-magnetic particles based on their mass density. The MDS process can separate more than two types of particles in a single process step, thereby distinguishing it from other separation techniques using a ferrofluid. The authors are currently constructing a superconducting MDS demonstrator. Ideally, the gradient of the magnetic field magnitude should change only with the distance above the magnet but remain constant in a horizontal plane. In principle, such an ideal field profile can be generated with an infinite harmonic sheet current. In practice, edge effects appear due to the necessity of using a finite number of coils. These cause a horizontal component in the field gradient and also change the vertical component. We compare the vertical magnetic field gradient of various coil layouts to see which configuration performs best. To facilitate ease of production, the analysis is restricted to flat racetrack coils. The main result is that the specific shape of a racetrack coil has a larger influence on the vertical gradient than the number of coils. The feed particles need to be pushed through the separation chamber from the insertion to the collection point. One option to realize this is to use an MDS setup in which the magnet is inclined with respect to the horizontal plane. This tilting results in a horizontal magnetic force component, which drives feed particles through the fluid bed. We show that a three-coil layout provides the largest usable fluid bed depth for a wide range of tilt angles.
\end{abstract}

Index Terms-Edge effects, ferrofluid, magnet, magnetic density separation (MDS), magnetic field gradient, racetrack coil, superconductor.

\section{INTRODUCTION}

$\mathbf{M}$ AGNETIC density separation (MDS) ideally requires a vertical gradient of the magnetic field magnitude that changes only with the distance perpendicular to the magnet but is constant in the parallel direction [1]. By immersing nonmagnetic shredded feed particles in a saturated ferrofluid that is subjected to such a vertical field gradient, particles with different mass densities move toward different equilibrium heights above the magnet. This recycling technology, thus, allows to separate feed particles based on mass density, e.g., for recycling purpose [1], [2]. A major advantage of the MDS technology compared to these other types of magnetic separation is its ability to separate multiple density components in a one-step process.

Currently, the University of Twente is constructing a superconducting NbTi-based magnet system that will be installed in the first superconducting MDS demonstrator MDS setup. The required magnetic field profile is different from other superconducting systems that have been developed for a variety of other separation systems [3]-[7], and thus, the optimum electromagnet configuration for MDS will also be different. Specifically, the gradient of the magnetic field strength should ideally be one-directional since field variations in multiple directions tend to re-mix the feed stream. The "ideal" current layout generating such a field is derived in a separate

Manuscript received February 14, 2021; revised April 19, 2021; accepted May 3, 2021. Date of publication May 13, 2021; date of current version July 20, 2021. Corresponding author: J. J. Kosse (e-mail: j.j.kosse@utwente.nl).

Color versions of one or more figures in this article are available at https://doi.org/10.1109/TMAG.2021.3080178.

Digital Object Identifier 10.1109/TMAG.2021.3080178 paper [12], which also explores the relation between particle equilibrium height, ferrofluid nanoparticle concentration, and magnetic field gradient. Building further upon the main conclusions from Kosse et al. [12], here, we focus on its practical implementation with the aid of a finite set of coils.

Due to larger design space and higher field strength, superconducting electromagnets enable an enhanced separation resolution and allow to use ferrofluids with a lower nanoparticle concentration compared to the currently employed permanent magnet (PM) technology [1] in MDS. The first superconducting MDS demonstrator will separate shredded electronic material, an application that is difficult with PM due to the wide range of mass densities involved. The magnet consists of three NbTi racetrack coils, conduction-cooled below $4.5 \mathrm{~K}$. The peak field on the magnet is $5.2 \mathrm{~T}$, and the operating current is $300 \mathrm{~A}$.

This demonstrator magnet will generate an average magnetic field value of $2 \mathrm{~T}$ at the bottom of the fluid bed, over a $0.9 \mathrm{~m} \times 1 \mathrm{~m}$ planar surface. The field magnitude decays exponentially with distance $z^{\prime}$ from the magnet as $\exp \left(-10.47 z^{\prime}\right)$. Compared to the more rapid decay of stateof-the-art PM-based MDS systems [8], [9], this increases the separation resolution by a factor of 2.5 . The usable fluid bed depth for the NbTi magnet is about $0.3 \mathrm{~m}$, whereas state-of-the-art PM systems are limited to fluid bed depths around $0.12 \mathrm{~m} \mathrm{[2].} \mathrm{The} \mathrm{usable} \mathrm{fluid} \mathrm{bed} \mathrm{dimensions} \mathrm{are}$ defined both by the desire for ferrofluid saturation, i.e., a background magnetic field strength of over $0.1 \mathrm{~T}$ [13], and a suitable magnetic field profile, as discussed in this work. While cleaning the feed particles after separation a fraction of the ferrofluid is lost, which constitutes a significant cost factor in 
this technology [10], the possibility to reduce the nanoparticle concentration, due to the stronger magnetic field [12], thus, lowers operational expenditure. For the separation of electronic material, the NbTi-based demonstrator allows a reduction in ferrofluid nanoparticle concentration of around $25 \%$.

An electromagnet necessarily comprises a finite number of coils. While the mathematical abstraction of a harmonic current distribution on an infinite plane produces the "ideal" (exponentially decaying) field [12], any practical magnet design requires truncation, which introduces edge effects. In this article, we consider how these effects influence the design of the magnet system.

Specifically, the focus is on how the coil shape and the precise number of coils in a superconducting MDS magnet influence the importance of the edge effects. For the treatment of electronic waste, a three-coil design is argued to be optimally explained by considering how the specifics of particle separation are affected by the shape and magnitude of the vertical and horizontal magnetic gradients. Similar considerations can also be made to find the optimum number of coils for future superconducting MDS systems designed for different feed streams.

In Section II, the specific method used to drive feed particles across the separation chamber, referred to as magnetdriven MDS, is presented. In Section III-A, different ways are discussed to approximate the ideal magnetic field shape for MDS with racetrack-type coils. The smoothness of the vertical magnetic field gradient produced by various numbers and types of coils is considered, showing that the desired vertical magnetic field gradient determines the specific shape of the racetrack coil. Finally, in Sections III-C and III-D, horizontal components of the magnetic field gradient are considered. The impact of these components depends on the method used to move feed particles across the separation chamber. In a magnet-driven MDS process, in which the set of coils is placed at an angle to the horizontal, the optimum number of coils is three, irrespective of the tilt angle.

\section{MAGnet-Driven MDS}

There are two qualitatively different strategies to drive the feed particles to move from one side of the separation chamber to the other.

The first method introduces a flow in the ferrofluid that drags the particles along. Characteristic flow speeds range from 0.1 to $0.3 \mathrm{~m} / \mathrm{s}$ [2]. In such a fluid-driven system, the minimum required coil number follows from the chosen flow speed and from the requirement that the feed particles are given sufficient time to reach their equilibrium heights. This time can be calculated by solving the trajectories of the feed particles numerically.

The second method introduces a horizontal component of the magnetic force to push the particles from their insertion point in the fluid toward the separator blades. This horizontal component can be created by placing the magnet at an angle with respect to the horizontal plane [11]. We refer to such a tilted MDS system as magnet-driven.

Fig. 1 shows a schematic where the magnet is rotated around the $y$-axis, introducing an angle $\alpha$ between the $x$ - and $x^{\prime}$-axes (or between $z$ and $z^{\prime}$ ). Since the ferrofluid pushes the feed

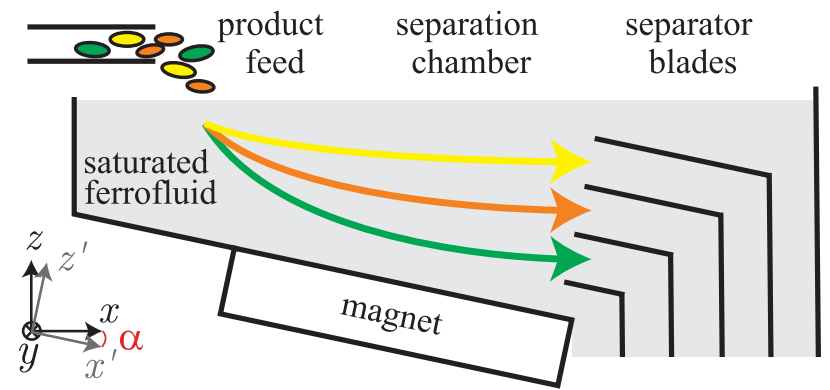

Fig. 1. Schematic of a magnet-driven MDS system. Non-magnetic feed particles are immersed in a ferrofluid that is attracted to a magnet. When the magnet is tilted at an angle $\alpha$ with respect to the horizontal, the combination of vertical forces on the feed particles (gravity, buoyancy, and effective repulsion by the magnet) dictates their equilibrium height, whereas the horizontal component of the magnetic repulsion pushes them toward the separator blades. After separation, the particles are collected by a conveyor belt that runs out of the sketched plane. Also indicated are the coordinate systems used in this article: $z$ is the vertical direction, while $z^{\prime}$ indicates the normal distance to the magnet surface.

particles away from the magnet in the $z^{\prime}$-direction, due to the fluid-magnet attraction, the force on a particle acquires a horizontal $x$-component when the magnet is tilted. Thus, the particles do not rely on a fluid flow in order to arrive at the collection points. This allows for a better separation resolution since turbulence is reduced [11].

Since the NbTi MDS demonstrator is magnet-driven, the focus in this article is on the tilted configuration. This is especially important in the discussion of the horizontal component of the magnetic field gradient. All considerations regarding the vertical component are also relevant for fluiddriven systems. To answer the question of which practical magnet configuration is best suited for a magnet-driven MDS, several magnet layouts are introduced and compared to each other.

\section{Potential Coil Layouts for MDS}

The ideal magnetic field configuration $H\left(x^{\prime}, y, z^{\prime}\right)$ for MDS has a gradient $\nabla H$ of its magnitude that only changes with distance $z^{\prime}$ from the magnet but remains constant parallel to it in the $x^{\prime} y$ plane. Such a magnetic field can be generated [12] by a periodic sheet current $K$ on an infinite $\left(x^{\prime}, y\right)$ plane of the form [see Fig. 2(b)]

$$
\boldsymbol{K}\left(x^{\prime}\right)=K_{0} \cos \left(\frac{2 \pi}{\lambda} x^{\prime}\right) \hat{\boldsymbol{y}}
$$

where $K_{0}$ is the magnitude of the sheet current and $\lambda$ is its periodicity. The resulting magnetic field above ${ }^{1}$ the sheet has a magnitude $H$ that decays exponentially with the distance $z^{\prime}$ from the sheet but does not depend on $x^{\prime}$ or $y$

$$
H\left(z^{\prime}\right)=\frac{K_{0}}{2} \exp \left(-\frac{2 \pi}{\lambda} z^{\prime}\right)
$$

Note how the periodicity of the current distribution $\lambda$ dictates the exponential decay length of the magnetic field magnitude.

\footnotetext{
${ }^{1}$ We assume that the feed particles have a higher mass density than the ferrofluid. Separation of feed streams with a lower mass density, e.g., certain plastics, is possible by placing the magnet above the fluid, pushing the particles into it [2].
} 


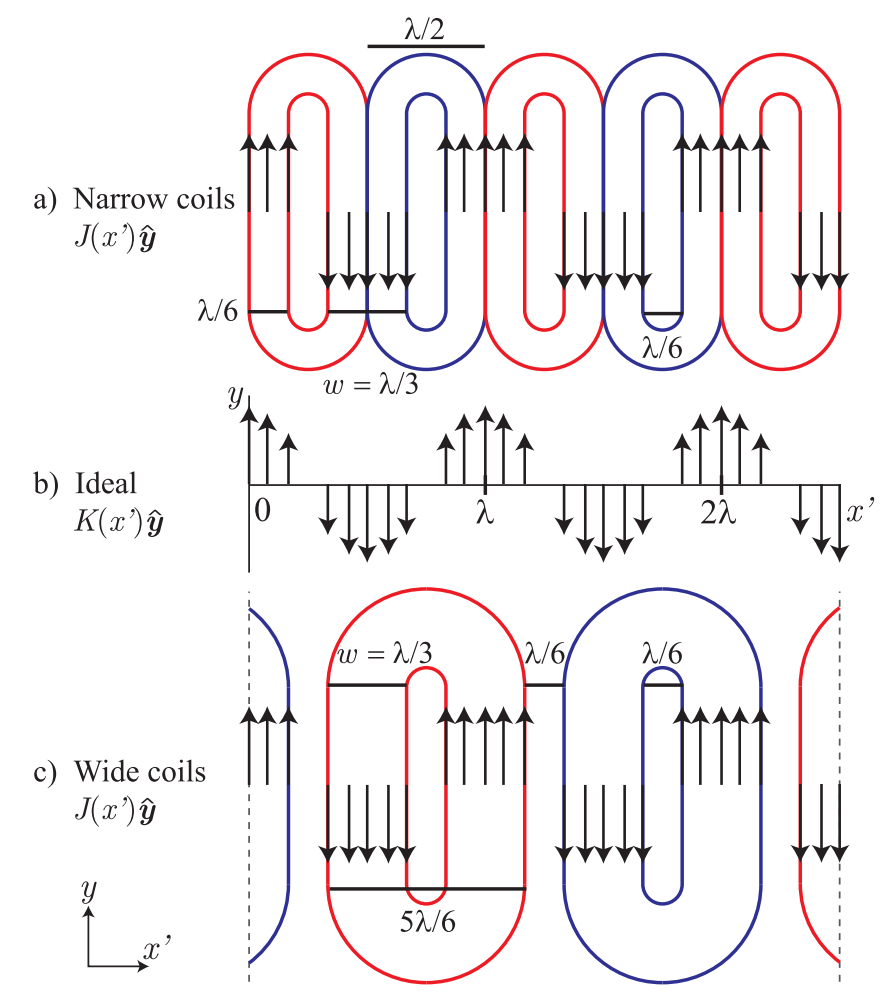

Fig. 2. Top view of coil layouts showing the ideal sheet current distribution $K$ for MDS (b) and two different methods (a and c) to approximate this sheet current with racetrack coils with rectangular cross-section that carry a uniform current density $J$. The leg width $w$ of the rectangular sections ideally is set to $\lambda / 3$. The first method (a) uses coils with a leg width $\lambda / 6$ and total width $\lambda / 2$ positioned directly adjacent to each other. The current direction (black arrows) in touching sections is the same. The second method (c) uses coils with a leg width $\lambda / 3$ and a coil width $5 \lambda / 6$, spacing them $\lambda / 6$ apart. Note that the layout of the semi-circular end sections can be optimized by segmenting them, in order to reduce the peak magnetic field in the conductor.

For the demonstrator magnet, $\lambda$ is chosen to be $0.60 \mathrm{~m}$. The difference in equilibrium height between two particles with different mass densities - and, hence, the separation resolutions-scales linearly with $\lambda$ [12]. The value of $0.60 \mathrm{~m}$ corresponds to the desired resolution for the separation of electronic waste.

\section{A. Practical Layouts of Racetrack Coils}

A practical approximation of such an idealized harmonic current distribution can be created with racetrack coils, placed in such a way that the current distribution varies stepwise with the $x^{\prime}$-coordinate. Preferably, the width $w$ of the racetracks' straight sections is set to $\lambda / 3$ [12] (see Fig. 2). In this way, the most dominant - unwanted-harmonic is suppressed. There are two straightforward ways to realize such a current distribution.

1) Racetracks coils, each with a total width of $\lambda / 2$ and a "leg" width $\lambda / 6$, are positioned directly next to each other in the $x^{\prime}$-direction [see Fig. 2(a)]. The current in adjacent legs flows in the same direction. We refer to this type of racetrack as a narrow coil.

2) Racetrack coils with a total width $5 \lambda / 6$ and a leg width $\lambda / 3$ are positioned with periodicity of $\lambda$ in the $x^{\prime}$-direction [see Fig. 2(c)]. This type of coil is referred to as a wide-coil. A downside of using such wide coils is that more material is needed to make the semicircular end sections. Also, the extra width of a coil for a given value of $\lambda$ can present more difficulties in coil winding.

In the remainder of this article, we focus on the magnetic field above the straight sections of the racetrack coils, not considering the semicircular parts. This is justified because the fluid bed is located above the straight sections of the coils.

The main questions addressed in this article are the optimum number of coils for an (angled) MDS magnet and whether the coils have to be of the wide- or narrow-variant. The questions are answered by focusing on two quantities.

1) The vertical component of the gradient of the magnetic field magnitude, $\partial H / \partial z$, which determines the equilibrium height of feed particles. Its variation with the horizontal coordinate $x$ influences the smoothness of particle trajectories.

2) The horizontal component of this gradient in the direction of movement, $\partial H / \partial x$. This component affects the horizontal movement of feed particles relative to the fluid, speeding them up or slowing them down.

With the different coil layouts introduced, Sections III-B and III-C will focus on the 2-D magnetic field profiles in the $x^{\prime} z^{\prime}$-cross sections (see Fig. 1) that coincide with the symmetry plane of the coils in the $y$-direction (see Fig. 2). After determining the best-performing configuration, 3-D simulation results are presented in Section III-D to argue that this $x^{\prime} z^{\prime}$ plane is representative for the entire fluid bed above the straight sections of the coils. It is obvious that the 2-D simulations do not provide details on the component of the gradient in the $y$-direction.

For the narrow-coil family, configurations that are made up of one to five coils are investigated. For the wide coils, up to three coils are considered. These eight different configurations are shown in Fig. 3, along with the magnetic field profiles above the coils that they produce.

The truncation of the periodic current distribution in the $x^{\prime}$-direction to a finite number of (half) periods changes the magnetic field compared to the ideal solution [see (2)]. Maybe, the most striking difference is the appearance, for some configurations, of local zeroes in the magnetic field. In the vertical direction, such nodes tend to compress different-density feed particles together, whereas, in the horizontal direction, they slow particles down. Both effects need to be minimized.

To compare the various configurations, we first consider the shape of the vertical magnetic field gradient.

\section{B. Variation in Vertical Magnetic Field Gradient}

In order to determine the optimal coil configuration for MDS, it is key to have a relevant criterion for magnetic field "quality." Ideally, the gradient of the magnetic field magnitude $\nabla H$ only has a vertical component that decays with the distance to the magnet. Variation of the vertical magnetic field gradient in the horizontal plane is to be suppressed as it causes the particle trajectory to "wiggle" due to a changing equilibrium height, which may lead to incomplete 

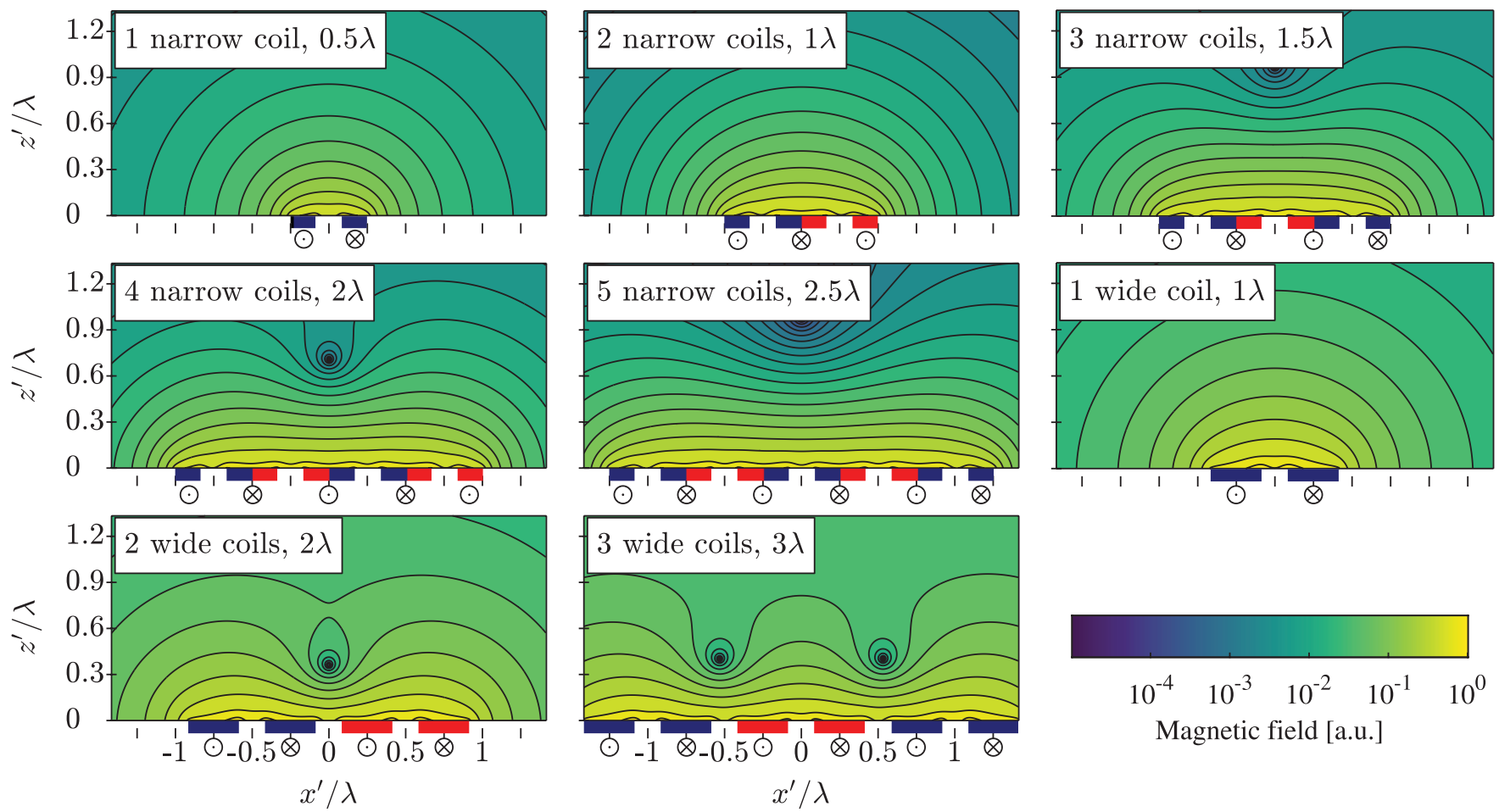

Fig. 3. Logarithm of the magnetic field magnitude $(\log H)$ above the surface of magnet systems consisting of varying numbers and types of coils. $x^{\prime} z^{\prime}$-cross sections at the mid-plane in the $y$-direction are shown. The racetrack coils are indicated alternately in red and blue. The small circles below the winding packs indicate the current direction (in the $\pm y$-direction). The origin of the $z^{\prime}$-axis is placed at the top surface of the coils. The magnetic field strength is normalized with the maximum value in the fluid bed across all considered configurations.

separation or even re-mixing. The demand for a ripple-free vertical-gradient can be expressed as ${ }^{2}$

$$
\frac{\partial^{2} H}{\partial x^{\prime} \partial z^{\prime}}=0
$$

Essentially, different magnet configurations can be compared by assessing how much they deviate from this condition. First, a rough qualitative analysis is made of how well different magnet configurations meet criterion (3). Then, for a more detailed comparison, a quantitative performance metric is formulated.

In Fig. 4, contour lines of the vertical magnetic gradient $\left|\nabla_{z} H\right|$ are plotted for the eight configurations that were also presented in Fig. 3. The lines can also be interpreted as the equilibrium heights of feed particles with different mass densities. Around the zero-field nodes, rings can be observed where $\nabla_{z} H=0$. At these locations, different-density particles will be pushed together, which is unwanted. At the first sight, the three-, four-, and five narrow-coil layouts seem promising since their contour lines look relatively flat and no zero-field nodes appear in the fluid bed.

To make this conclusion more quantitative, we consider a section of the fluid bed with a rectangular shape bound by $\left[z_{1}^{\prime}, z_{2}^{\prime}\right]$ and $\left[x_{1}^{\prime}, x_{2}^{\prime}\right]$. As a performance metric for fluctuations in the vertical field gradient at a given distance $z^{\prime}$ above the magnet, the relative standard deviation RSD is used. It is the standard deviation of $\left|\nabla_{z} H\right|$ divided by its mean value

\footnotetext{
${ }^{2}$ The other requirement, $\left(\partial^{2} H / \partial y \partial z^{\prime}\right)=0$, is automatically fulfilled because the $y$-symmetry plane is considered.
}

in the $x^{\prime}$-range of interest $\left[x_{1}^{\prime}, x_{2}^{\prime}\right]$

$$
\begin{aligned}
& \operatorname{RSD}\left(\left[x_{1}^{\prime}, x_{2}^{\prime}\right], z^{\prime}\right) \\
& =\frac{\sigma\left(\left[x_{1}^{\prime}, x_{2}^{\prime}\right], z^{\prime}\right)}{|\mu|\left(\left[x_{1}^{\prime}, x_{2}^{\prime}\right], z^{\prime}\right)} \\
& =\frac{\left[\frac{1}{x_{2}^{\prime}-x_{1}^{\prime}} \int_{x_{1}^{\prime}}^{x_{2}^{\prime}}\left(\nabla_{z} H\left(x^{\prime}, z^{\prime}\right) \mathrm{d} x^{\prime}-\mu\left(\left[x_{1}^{\prime}, x_{2}^{\prime}\right], z^{\prime}\right)\right)^{2} \mathrm{~d} x^{\prime}\right]^{0.5}}{\left|\mu\left(\left[x_{1}^{\prime}, x_{2}^{\prime}\right], z^{\prime}\right)\right|}
\end{aligned}
$$

where $\mu\left(\left[x_{1}^{\prime}, x_{2}^{\prime}\right], z^{\prime}\right)$ is the mean of the vertical field gradient $\nabla_{z} H$ at the height $z^{\prime}$, evaluated over the same $x^{\prime}$-range

$$
\mu\left(\left[x_{1}^{\prime}, x_{2}^{\prime}\right], z^{\prime}\right)=\frac{1}{x_{2}^{\prime}-x_{1}^{\prime}} \int_{x_{1}^{\prime}}^{x_{2}^{\prime}} \nabla_{z} H\left(x^{\prime}, z^{\prime}\right) \mathrm{d} x^{\prime} .
$$

The RSD value is shown in Fig. 5 as a function of the normalized distance $z^{\prime} / \lambda$ to the surface of the coils. Configurations with two or three wide coils show large variations at distances $z^{\prime} / \lambda \gtrsim 0.3$, due to the presence of zero-field nodes in this area, as also obvious in Figs. 3 and 4.

All configurations show large variations near the coil surface $\left(z^{\prime} / \lambda \lesssim 0.05\right)$. These are a direct consequence of the discrete "steps" in the current distribution $J$ of the racetrack system as opposed to the smooth harmonic ideal current distribution $K$ (see Fig. 2) [12]. ${ }^{3}$ Note that a minimized fluid-coil distance is desired since, for a given number of ampere-turns,

\footnotetext{
${ }^{3}$ The variations that result from using a rectangular current distribution instead of the ideal harmonic distribution can be reduced by using thicker coils [12]
} 

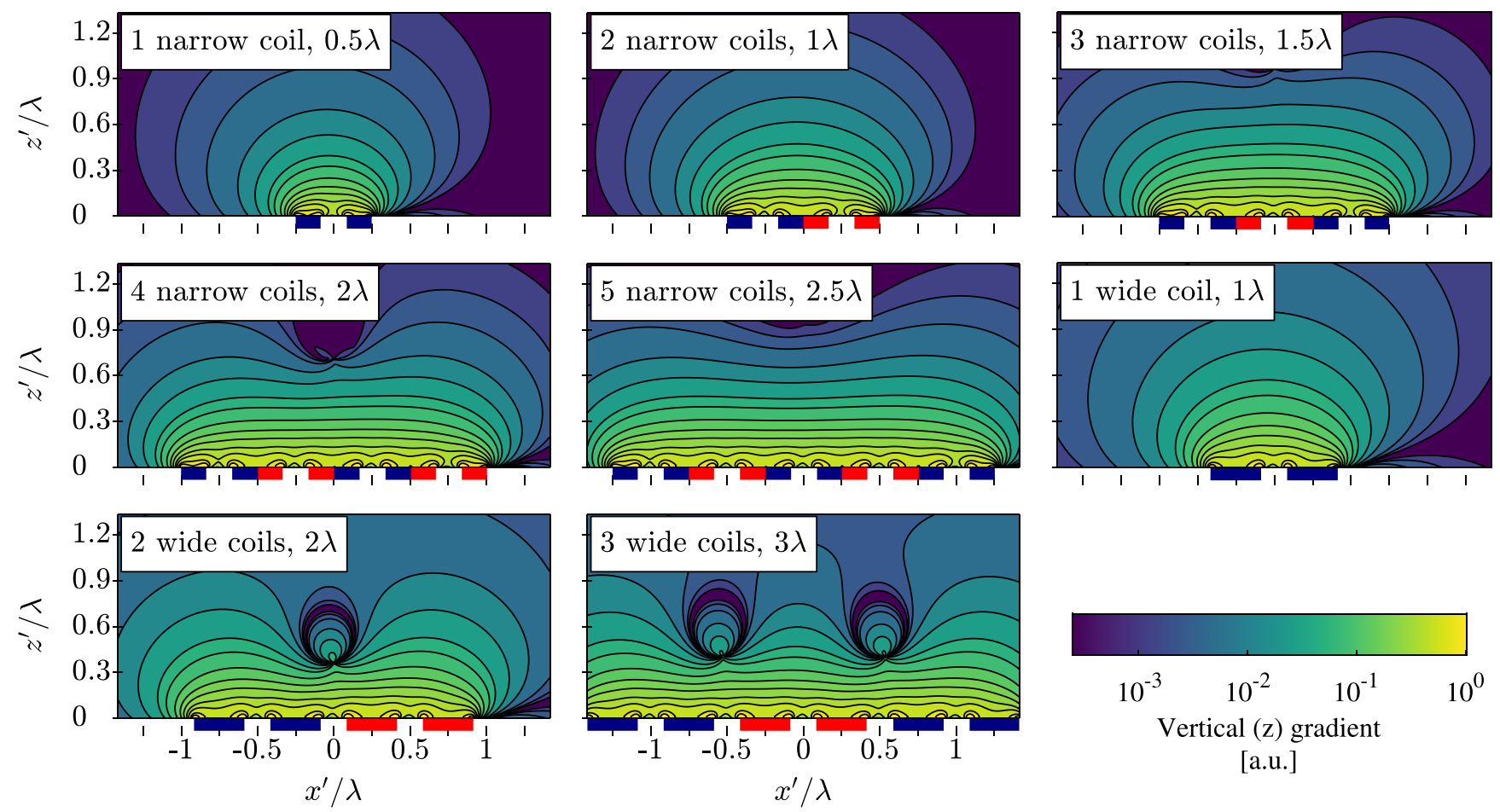

Fig. 4. Logarithm of the magnitude of the vertical $(z)$ magnetic field gradient, $\log \left|\nabla_{z} H\right|$, above the surface of the sets of coils. Like Fig. 3, the plots show the $x^{\prime} z^{\prime}$-cross section at the $y$-symmetry plane. The coils are indicated in red and blue. The magnetic field gradient is normalized with its maximum value encountered across all considered configurations. The asymmetry in the $x^{\prime}$-coordinate is due to the $\alpha=12^{\circ}$ angle between the $z$ - and $z^{\prime}$-axes (see Fig. 1 ).

this yields a higher vertical magnetic field gradient in the fluid bed and, thus, allows to separate heavier feed streams or-as discussed in the introduction-to use more dilute ferrofluids.

The impact of the variations near the coils on the separation performance depends on the coil-to-fluid distance. For the $\mathrm{NbTi}$ demonstrator magnet under development, with $\lambda=$ $0.60 \mathrm{~m}$, this area with large fluctuations is situated within the first $30 \mathrm{~mm}$ from the coils, i.e., inside the cryostat (the distance between the coils and the fluid is $50 \mathrm{~mm}$ in this system). However, if one aims to use this region for the separation process (for example, when one manages to maintain the same coil-to-fluid distance with a larger $\lambda$ magnet), it can be advantageous to use "notched" coils with a more complex shape that more closely resembles the ideal current distribution [12].

At intermediate distances from the magnet $\left(0.05 \lesssim z^{\prime} /\right.$ $\lambda \lesssim 0.6$ ), Fig. 5 shows how, for the narrow-coil layouts, the deviations do not differ much from one configuration to the other. Narrowing the considered range $\left[x_{1}^{\prime}, x_{2}^{\prime}\right]$ results in lower RSD values - since edge effects then play a smaller role — and, thus, in a downward shift of all curves. However, the same qualitative behavior remains. Thus, based on the "quality" of the vertical field gradient, we can draw two conclusions: 1) the wide-coil family is not practical if the useful fluid bed depth is to be larger than $\lambda / 4$ and 2) in principle, any reasonable number of narrow coils may be used. To determine an optimum number of narrow coils, the horizontal component of the field gradient needs to be considered as well. This is the focus of Section III-C.
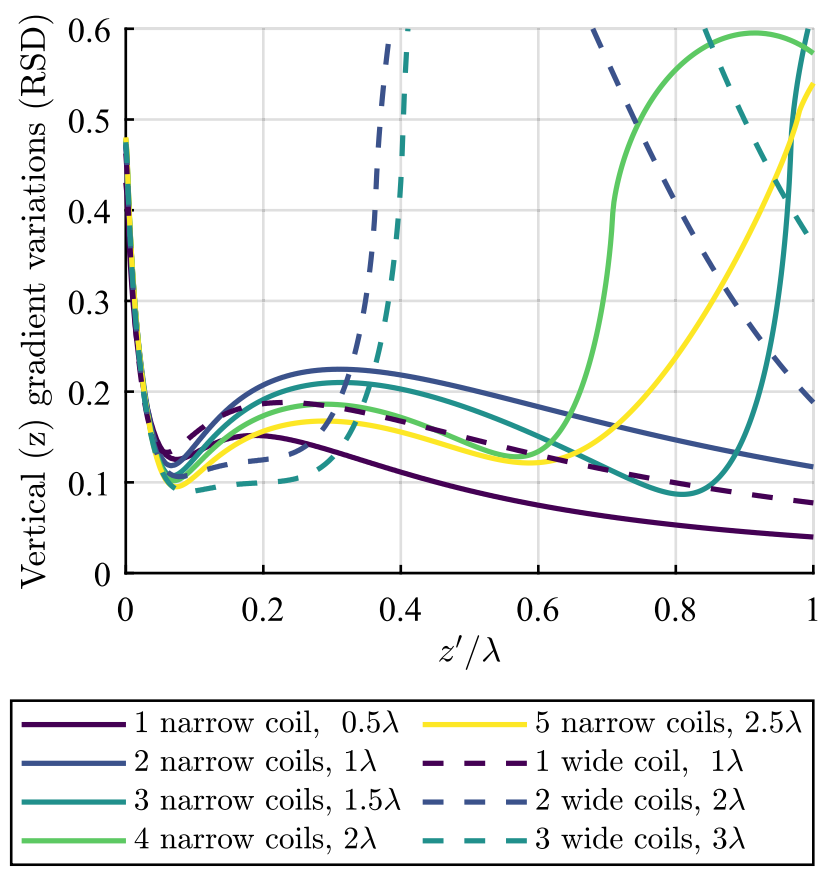

Fig. 5. Normalized vertical $z$-gradient variation, as defined by (4b), as a function of distance $z^{\prime}$ from the top surface of the plane of coils. The fluctuations in the vertical magnetic field gradient at each height are evaluated using a horizontal coordinate between the leftmost and the rightmost $x^{\prime}$-coordinate of the coils. The winding pack thickness for these examples is $\lambda / 12$.

\section{Effect of Tilting the Plane of Coils}

For the NbTi demonstrator magnet, a tilt angle $\alpha$ of $12^{\circ}$ was selected (see Fig. 1). If $\alpha$ is too large, the horizontal 


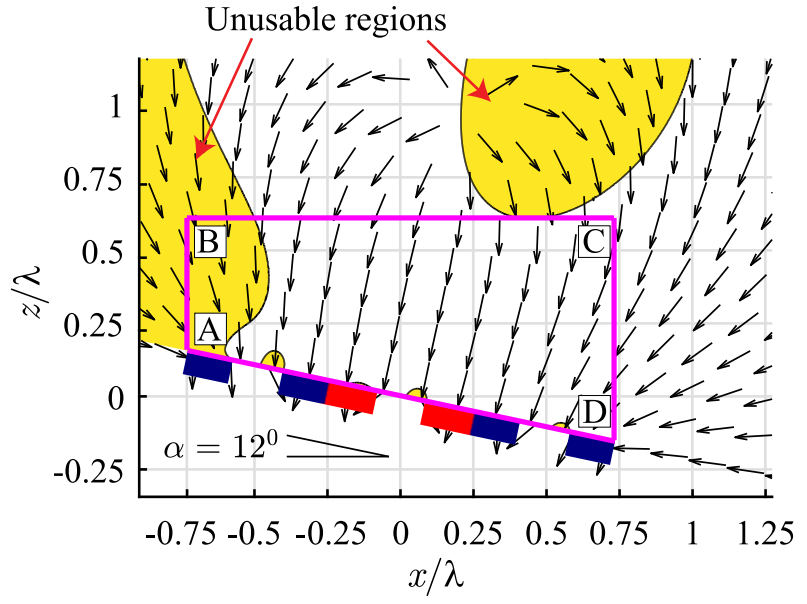

Fig. 6. Direction of the gradient in the magnetic field magnitude (black arrows) above a tilted three-coil magnet (red and blue rectangles). Areas where the gradient has a positive $x$-component are indicated in yellow. These areas are unusable for MDS since feed particles get pushed in the (wrong) negative $x$-direction there. This particular example shows coils tilted at $12^{\circ}$, but qualitatively similar observations can be made for all magnet-driven systems.

component of the force on the feed particles is too high so that they have not arrived yet at their vertical equilibrium height by the time they reach the separator blades [11]. Also, a too high travel speed may reduce the separation resolution due to fluid-dynamical lift forces on asymmetric feed particles. On the other hand, when $\alpha$ is too small, the MDS throughput is suboptimal since the particles' speed in the $x$-direction is smaller. The choice for a $12^{\circ}$ angle is based on the experience of the end-user of the PM systems. Preferably, the angle is between $5^{\circ}$ and $20^{\circ}$ for practical applications [11].

Another important issue is whether the horizontal component of the magnetic field gradient everywhere points in the correct direction in the fluid bed. This will determine whether all feed particles get pushed all the way across the separation chamber to the separator blades.

Fig. 6 shows the direction of the magnetic field gradient in the ferrofluid above a three-coil system that is tilted at $12^{\circ}$. Areas where the $x$-component of the gradient is positive provide difficulties for the particle flow since the magnetic force on the ferrofluid effectively pushes the feed particles in the opposite direction. Unlike in a fluid-driven system, the fluid does not drag the particles across these regions, and thus, these areas are unusable. This dictates a second demand on the magnetic field in the separation chamber, in addition to (3), namely

$$
\frac{\partial H}{\partial x}<0 .
$$

The regions that do not meet this requirement are highlighted in Fig. 6. The leftmost colored region limits where the feed particles can be fed into the fluid bed. The colored region more to the right limits the usable fluid bed depth and, thus, the separation performance of feed products with lower mass density. To compare this usable depth for different magnet angles and different numbers of coils, we define a vertical distance $\mathrm{AB}$ between the top of the tilted magnet and the

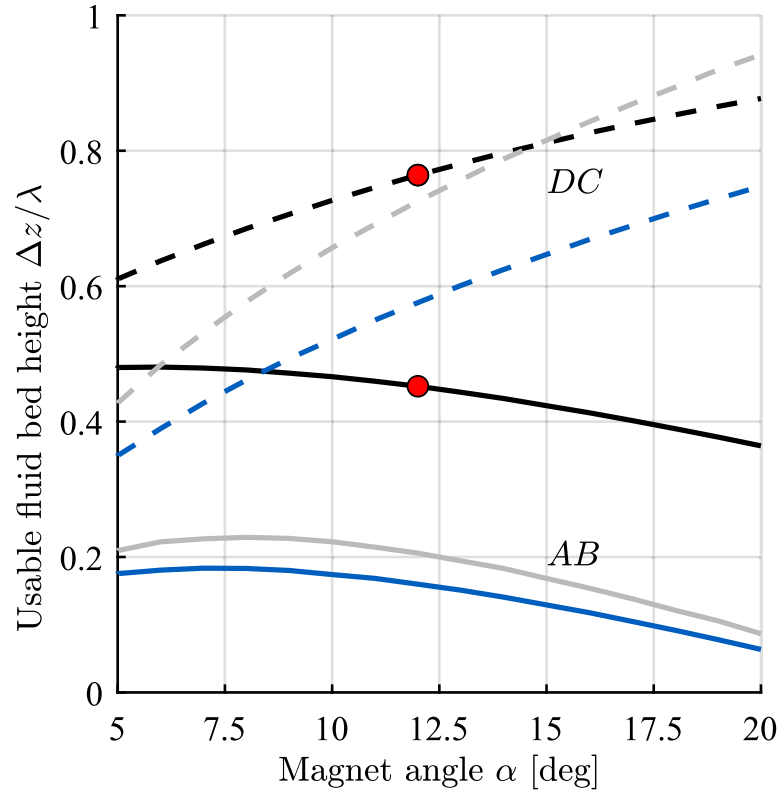

$A B, 3$ narrow coils $---D C, 3$ narrow coils
$A B, 4$ narrow coils $---D C, 4$ narrow coils
$A B, 5$ narrow coils $---D C, 5$ narrow coils
NbTi demonstrator

Fig. 7. Usable vertical distance between the surface of the coils and the fluid surface, as defined in Fig. 6, plotted against the tilt angle $\alpha$. Different colors correspond to different coil numbers, all of the narrow-coil family (see Fig. 2). Solid lines denote the vertical distance $A B$ between the left-hand side of the magnet and the unusable area. Dashed lines denote the vertical distance DC between the extraction side of the magnet and the problem area. To determine the actual useful fluid depth, one needs to subtract the distance between the coils and the cryostat surface, which is roughly $50 \mathrm{~mm}$ (or $\lambda / 12$ ) for the NbTi three-coil magnet design (indicated with the red symbols).

problem region, and DC between the rightmost location of the magnet and the problem area. These distances are also indicated in Fig. 6.

$\mathrm{AB}$ and $\mathrm{DC}$ are shown as functions of the tilt angle $\alpha$ in Fig. 7 for three to five narrow coils. DC is indicative of the obtainable separation resolution since it determines the range over which separator blades (see Fig. 1) can be spaced. A low $\mathrm{AB}$ value is not useful since it implies that feed particles with a relatively low mass density are pushed to the ferrofluid surface after being inserted in the fluid bath and only start to separate once they have been pushed further along the fluid bed. Thus, a large value of both AB and DC is desired. Fig. 7 clearly illustrates how the three-coil layout provides the largest usable depth of the fluid bed for a wide range of tilt angles. This is the main reason that three coils placed at a $12^{\circ}$ angle were selected for the final layout of the $\mathrm{NbTi}$ demonstrator system.

Not only the vertical distance is of interest but also the effective fluid bed volume. In this respect, the area enclosed by $\mathrm{ABCD}$ is taken as a rough figure of merit. A large value of $A B C D$ area is beneficial as it minimizes the effect of interactions between feed particles due to the extra available space. The result is a higher separation resolution and throughput. The $\mathrm{ABCD}$ area as a function of the tilt angle is shown in Fig. 8. The three-coil layout provides a larger 


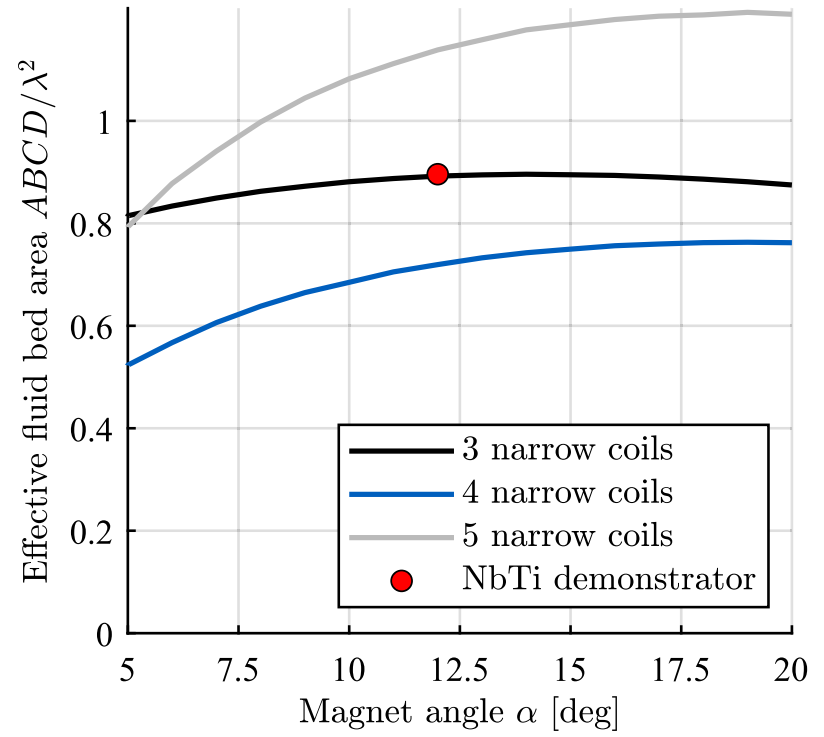

Fig. 8. Scaled effective fluid bed area $\mathrm{ABCD} / \lambda^{2}$, as defined in Fig. 6, versus the magnet tilt angle $\alpha$. Different colors correspond to layouts with different numbers of coils, all of the narrow family.

separation volume than the four-coil one. The five-coil layout performs similar to the three-coil one at lower $\alpha$ values. At larger angles, the five-coil configuration provides a larger separation volume, though always below a 5/3 ratio compared to the three-coil one. This means that the three-coil system provides the largest separation volume per coil, which can be an important consideration for MDS systems in which the coils contribute a significant factor to the expenditure over the lifetime of the system. Subtracting the part of this area inside the cryostat or subtracting the leftmost problem region in Fig. 6 does not change these conclusions.

The wide-coil configurations are omitted from this discussion since they generate regions with a positive horizontal component of the magnetic field gradient too close to the magnet to make a magnet-driven strategy possible.

\section{3-D Analysis}

In this section, a full 3-D magnetic field calculation of the three-narrow-coil solution made with COMSOL to verify that the conclusions drawn above based on 2-D calculations of the magnetic field profile are still valid when one approaches the semicircular "bends" at both ends of the racetrack coils (see Fig. 2) is presented. Fig. 9 shows contour lines of the vertical $z$-component of the gradient in three $x^{\prime} z^{\prime}$ planes corresponding to different $y$ values. The length of the straight sections of the racetrack coils is set to $1 \mathrm{~m}$. Note that this length determines the throughput of the MDS process. The plane at $y=0$ corresponds to the horizontal symmetry plane in Fig. 2, while the $y=0.5 \mathrm{~m}$ plane shows the transition between the racetrack coils' straight and semicircular sections. $\lambda$ is set to $0.60 \mathrm{~m}$ as in the NbTi demonstrator. The contour lines move closer to the coil surface for $y$ values further from the center of the coils, but these deviations are small enough $(\Delta z \lesssim 10 \%)$ to be correctable by slightly shaping the separator blades.

Another option would be to bend the winding packs themselves so that the distance between the ferrofluid and the coils

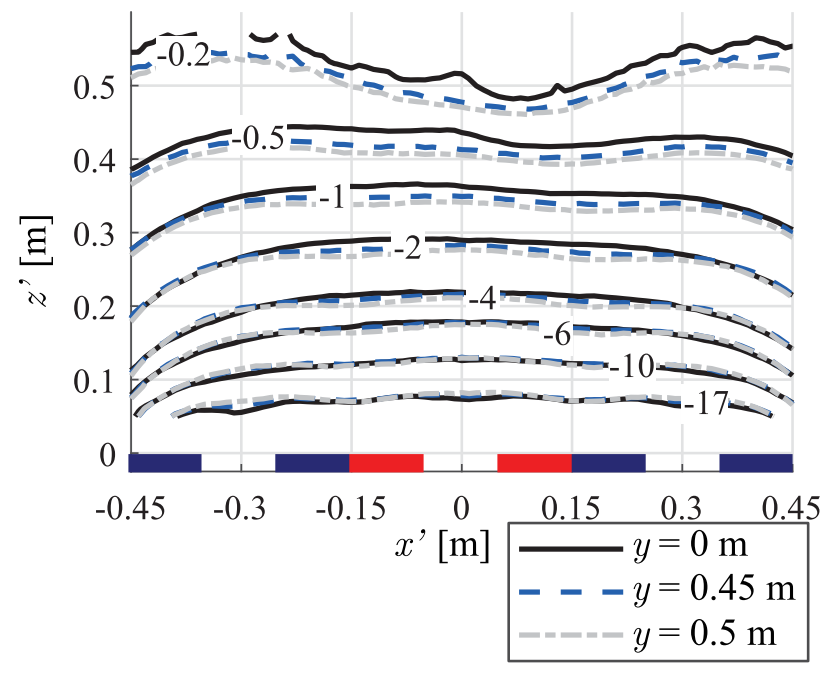

Fig. 9. Contour lines of the vertical $z$-component of the gradient $\mu_{0} \nabla_{z} H$ in $[\mathrm{T} / \mathrm{m}]$, for three vertical $x^{\prime} z^{\prime}$ planes located at different $y$-coordinates (indicated with different line styles). The example shown is for the $12^{\circ}$ angled $\mathrm{NbTi}$ demonstrator magnet consisting of three coils (shown in blue and red) with a winding pack thickness of $50 \mathrm{~mm}$. The length of the straight sections of the racetrack coils is $1 \mathrm{~m}$. The coordinate system used is defined in Fig. 1, and $z^{\prime}=0$ corresponds to the top surface of the coils. Each coil has 2230 turns and carries a current of $300 \mathrm{~A}$.

is slightly higher near the middle $(y=0)$, which would also reduce particle motion in the $y$-direction. However, for this first-of-its-kind demonstrator, the focus is on simplicity and ease of coil winding so that shaping the magnetic field by bending the coils was omitted. Also, this approach will be impractical for superconductors in the tape form whose performance suffers under so-called "hard-bending" [14].

As for the variation along the $y$-direction of the $x$-component of the gradient, the location and shape of the unusable regions shown in Fig. 6 do not change significantly when coming close to the racetrack coils' semicircular sections.

Another aspect of interest is the ratio $(\partial H / \partial y) /(\partial H / \partial x)$ between the two horizontal components of the magnetic field gradient. This ratio determines how much particles move along the $y$-direction toward the side of the fluid tank while traveling through the fluid bed in the $x$-direction and is shown in Fig. 10. The relative importance of the $y$-component gets stronger with increasing distance to the coils' surface.

Fig. 10 also shows a sketch of the coils. The bent coils' heads are split into two sections, which reduces the peak magnetic field in the bends from $119 \%$ down to $107 \%$ of the magnetic field in the straight sections. In Fig. 9, the effect of this modification is taken into account.

In principle, in a fluid-driven MDS system, the ferrofluid can flow in either the $x$ - or $y$-direction. However, the 3-D simulations show that, with a magnet-driven system tilted around the $x$-axis (see Fig. 2), the resulting component of the gradient is insufficient to push particles to the collections points. Note that the feed particles are driven in the $x$-direction (see Fig. 2), even if, in principle, for the fluid-driven MDS type, a fluid flow may be allowed either in the $x$ - or $y$-direction. More precisely stated, large ineffective areas appear with a positive $y$-component of the magnetic field gradient. 

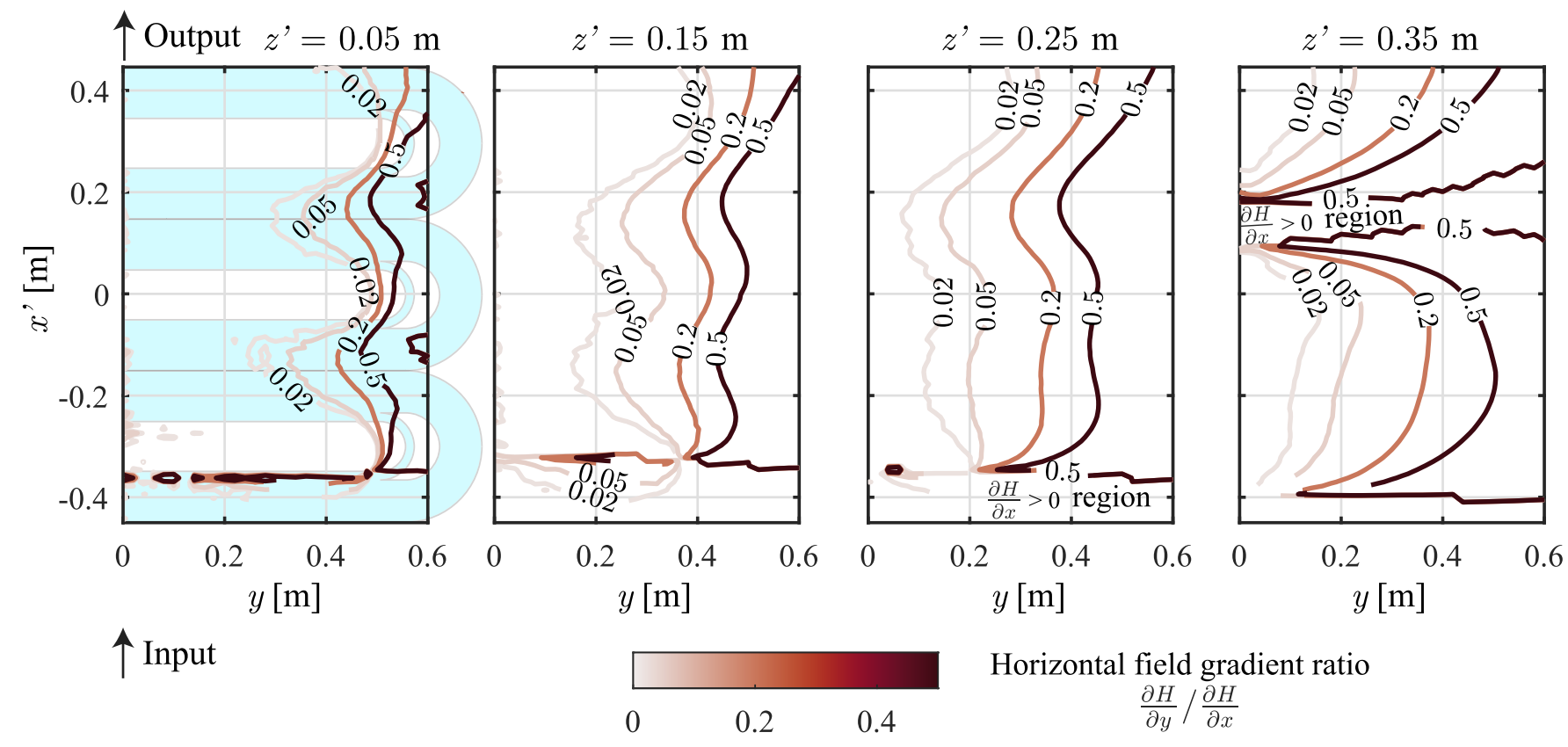

Horizontal field gradient ratio $\frac{\partial H}{\partial y} / \frac{\partial H}{\partial x}$

Fig. 10. Contour lines of the ratio between the horizontal components of the magnetic field gradient, evaluated in the $x^{\prime} y$ planes at different distances $z^{\prime}$ to the coil surface. The coils are included in the leftmost subplot. The $y$-component of $\bar{\nabla} H$ should be minimal, so as to minimize movement of particles toward the sidewalls. The relative importance of the unwanted $y$-gradient increases with increasing distance to the magnet. Feed particles have to be inserted into the ferrofluid at $x^{\prime}$ values larger than $-0.4 \mathrm{~m}$ due to the presence of a positive $x$-gradient region, as discussed in the context of Fig. 6 . The other problem region is visible at a distance $z^{\prime}$ of $0.35 \mathrm{~m}$ (the rightmost subplot).

TABLE I

Main Parameters of the NbTi Demonstrator Magnet RElevant to SEParation. The NARrow-CoIl VARiant Was INTRODUCED IN FIG. 2

\begin{tabular}{llll} 
Parameter & Symbol & Value & Unit \\
\hline Magnetic field at fluid bed & $\mu_{0} H_{\text {fluid }}$ & 2.0 & $\mathrm{~T}$ \\
Coil-fluid distance & & 50 & $\mathrm{~mm}$ \\
Characteristic periodicity & $\lambda$ & 0.60 & $\mathrm{~m}$ \\
Magnet tilt & $\alpha$ & 12 & degrees \\
Racetrack coil type & & narrow & \\
Number of coils & & 3 & \\
Coil width & $\lambda / 2$ & 0.30 & $\mathrm{~m}$ \\
Winding pack thickness & $d_{\text {coil }}$ & 50 & $\mathrm{~mm}$ \\
Coil straight section length & $L_{y}$ & 1.00 & $\mathrm{~m}$
\end{tabular}

In the previous discussion, the winding pack thickness was set to $50 \mathrm{~mm}$. This corresponds to the NbTi demonstrator, whose key properties are summarized in Table I. The system consists of three coils, each with a width of $0.3 \mathrm{~m}$ and a length of the straight sections of $1 \mathrm{~m}$. The splitting of the coil heads (to reduce the peak magnetic field) extends the length of the coils by $100 \mathrm{~mm}$, resulting in a total coil length of $1.4 \mathrm{~m}$ in the $y$-direction. The magnet generates a $2.0 \mathrm{~T}$ field at the bottom of the fluid bed [15].

\section{CONCLUSION}

For MDS, an electromagnet can be used to generate the required gradient in the magnitude of the magnetic field. This gradient ideally only has a component perpendicular to the magnet system. The main question discussed in this article was which practical coil configuration optimally generates such a required magnetic field profile. Magnet layouts were introduced with two possible types of racetrack coils, "wide" and "narrow," and configurations with a different number of coils were compared. The wider coils are unsuited due to the presence of zero-field nodes in the separation area.

When selecting the optimum number of coils for an MDS system, one needs to distinguish between the two different strategies that can be used to drive the feed particles through the fluid bed. One method is to tilt the magnet with respect to the horizontal plane and, thus, to create a horizontal component of the magnetic force.

The vertical distance between the magnet and "problem areas" in which this horizontal component has the wrong direction (i.e., pushes the particles back) depends on the number of coils and the tilt angle. This vertical distance determines the maximum usable fluid bed depth and, hence, the separation performance of an MDS system. For the first NbTi demonstrator magnet designed for the separation of electronic waste, an angle of $12^{\circ}$ is selected. We showed that a three-coil configuration is superior to a four- or five-coil-based system for a wide range of tilt angles.

The comparison between coils is based on a 2-D analysis of the magnetic field profile, and the selected final layout was verified with a more detailed 3-D analysis.

\section{ACKNOWLEDGMENT}

This work was supported in part by the Dutch Ministry of Economic Affairs. This research is part of the program 
Innovative Magnetic Density Separation (IMDS), which is supported by NWO, The Netherlands Organisation for Scientific Research, Domain Applied and Engineering Sciences.

\section{REFERENCES}

[1] E. J. Bakker, P. C. Rem, and N. Fraunholcz, "Upgrading mixed polyolefin waste with magnetic density separation," Waste Manage., vol. 29, no. 5, pp. 1712-1717, May 2009.

[2] B. Hu, "Magnetic density separation of polyolefin wastes," Ph.D. dissertation, Dept. Civil Eng. Geosci., Univ. Delft, Delft, The Netherlands, 2014.

[3] J. X. Jin et al., "A high gradient magnetic separator fabricated using bi-2223/Ag HTS tapes," IEEE Trans. Applied Supercond., vol. 9, no. 2, pp. 394-397, Jun. 1999.

[4] R. Gerber and M. Watmough, "A design of a superconducting split-coil open gradient magnetic separator," IEEE Trans. Magn., vol. MAG-21, no. 5, pp. 2053-2055, Sep. 1985.

[5] J. Iannicelli et al., "Magnetic separation of kaolin clay using a high temperature superconducting magnet system," IEEE Trans. Applied Supercond., vol. 7, no. 2, pp. 1061-1064, Jun. 1997.

[6] J. Boehm, R. Gerber, D. Fletcher, and M. R. Parker, "Deflection of weakly magnetic materials by superconducting OGMS," IEEE Trans. Magn., vol. MAG-24, no. 2, pp. 1674-1676, Mar. 1988.

[7] D. D. Jackson, "Processing of China clays using a commercial-scale, conduction-cooled superconducting magnetic separation system," IEEE Trans. Magn., vol. 49, no. 7, pp. 3438-3440, Jul. 2013.
[8] S. Serranti, V. Luciani, G. Bonifazi, B. Hu, and P. C. Rem, "An innovative recycling process to obtain pure polyethylene and polypropylene from household waste," Waste Manage., vol. 35, pp. 12-20, Jan. 2015.

[9] V. Luciani, G. Bonifazi, P. Rem, and S. Serranti, "Upgrading of PVC rich wastes by magnetic density separation and hyperspectral imaging quality control," Waste Manage., vol. 45, pp. 118-125, Nov. 2015.

[10] H. Gobel, Inventarisatie Scheidingstechnieken Harde Polyolefinen in Polypropyleen $(P P)$ en Polyethyleen $(P E)$, Mechelen, Belgium: The Public Waste Agency of Flanders (OVAM), 2009.

[11] P. C. Rem and P. Berkhout, "Splitter for magnetic density separation," International Patent No.WO 2017111583 A1, Jun. 29, 2017.

[12] J. J. Kosse, M. Dhallé, P. C. Rem, H. J. M. ter Brake, and H. H. J. ten Kate, "Fundamental electromagnetic configuration for generating one-directional magnetic field gradients," to be published.

[13] A. M. van Silfhout, H. Engelkamp, and B. H. Erné, "Colloidal stability of aqueous ferrofluids at 10 t," J. Phys. Chem. Lett., vol. 11, no. 15, pp. 5908-5912, Aug. 2020.

[14] H. S. Shin, M. J. Dedicatoria, J. R. C. Dizon, H. S. Ha, and S. S. Oh, "Bending strain characteristics of critical current in REBCO CC tapes in different modes," Phys. C, Supercond., vol. 469, nos. 15-20, pp. 1467-1471, Oct. 2009.

[15] J. J. Kosse, M. Dhallé, G. Tomás, P. C. Rem, H. J. M. ter Brake, and H. H. J. ten Kate, "Performance estimates of superconducting magnetic density separation," to be published. 